International Journal of Child, Youth and Family Studies (2012) 4: 396-408

\title{
YOUNG PEOPLE'S EXPERIENCES IN YOUTH-LED PARTICIPATORY ACTION RESEARCH FOR HIV/AIDS PREVENTION
}

\author{
Keiko Goto, Jennifer Tiffany, Gretel Pelto, and David Pelletier
}

\begin{abstract}
As part of a project in the UNICEF-initiated "What every adolescent has a right to know" (RTK) program, qualitative interviews were conducted with youth (13 to 21 years of age) who were reached through a youth-led participatory action research (PAR) project. The interviews were conducted with both urban and rural participants in a Caribbean country where potential exposure to HIV/AIDS represents a significant threat to young people's health. The purpose of the study was to examine how a truly youth organized PAR effort was perceived by the adolescents who were reached through the project. The results suggest that the central role played by youth researchers (the PAR community leaders) created a mode of participation that facilitated the collaboration of at-risk and marginalized youth. The type of engagement created was distinct from modes of participation fostered when adults alone worked with youth.
\end{abstract}

Keywords: youth, HIV/AIDS, participatory action research

Acknowledgements: This study was supported by the Mario Einaudi Center for International Studies, the Graduate School and the Division of Nutritional Sciences at Cornell University. The authors would like to thank the participants and staff of the UNICEF RTK initiative for their support.

Keiko Goto, Ph.D. (the corresponding author) is Associate Professor at the Department of Nutrition and Food Sciences, California State University, Chico, 400 West First Street, Chico, California, U.S.A., 95929-0002. Phone: (530) 898-6767, Fax: (530) 898-5586, E-mail: kgoto@csuchico.edu

Jennifer Tiffany, Ph.D. is Director of HIV/AIDS Education at Cornell University, Ithaca, New York, U.S.A., 14853. Phone: (607) 255-1942, Fax: (607) 255-8562, Email: jst5@cornell.edu

Gretel Pelto, Ph.D. is Graduate Professor at the Division of Nutritional Sciences, Cornell University, Ithaca, New York, 14853. Phone: (607) 255-2608, Fax: (607) 255-1033, E-mail: gp32@cornell.edu

David Pelletier, Ph.D. is Associate Professor of Nutrition Policy at the Division of Nutritional Sciences, Cornell University, 212 Savage Hall, Ithaca, New York, 14853. Phone: (607) 255-1086, Fax: (607) 255-1033, E-mail: dlp5@cornell.edu 
Practitioners and investigators in the field of health promotion frequently employ participatory approaches. There are various types of participatory approaches, such as participatory action research (PAR) (Kemmis \& McTaggart, 2000), community-based participatory research (CBPR) (Minkler \& Wallerstein, 2003), participatory rural appraisal (PRA) (Chambers, 1994), and participatory learning and action (PLA) (Blackburn \& Holland, 1998), all of which promote the participation of local people, communities, and organizations. PAR has been defined as "systematic inquiry with the collaboration of those affected by the issue being studied, for purposes of education and taking action or effecting change" (Green et al., 2003, p. 419). PAR is also employed to transform practitioners' theories and practices in particular local settings (Kemmis \& McTaggart, 2000). Research projects that involve interventions directed to young people increasingly promote youth participation in order to more effectively learn about and address issues that affect the lives and health of youth (Powers \& Tiffany, 2006; Maglajlic \& Tiffany, 2006).

This paper examines a specific aspect of participatory action research with youth, namely, the perceptions of the marginalized and at-risk youth participants who were reached through being involved in a youth-led project. The project was part of an initiative undertaken by the United Nations International Children's Emergency Fund (UNICEF) to address the challenge of preventing HIV/AIDS in young people. The program, entitled "What every adolescent has a right to know" (RTK), was initiated by UNICEF to promote the prevention of HIV/AIDS in youth and was implemented in 14 countries in various parts of the world (UNICEF, 2002). It sought to ensure that young people not only received accurate information, but were also empowered to make informed choices (UNICEF, 2002). Participatory action research was identified as an approach that would enable youth leaders to be involved in planning and implementing the research, and to bring in other adolescents from the community. As a technical partner, a Cornell University team, consisting of the four authors of this paper and other researchers, provided UNICEF with technical assistance regarding participatory action research (PAR) and the right to know (RTK) project.

This report is drawn from the experiences in one of the RTK country PAR projects in which youth peer educators (16 to 22 years old) from various previously existing youth organizations became PAR researchers. With support from adults, including project managers at youth-serving organizations, UNICEF, and the Cornell team, the youth researchers conducted PAR with other youth from the community - 13 to 21 years olds who were potentially "at risk" for HIV/AIDS and/or who were not reached by other youth programs. In this paper, participants in the latter group are referred to as "sharing team members" whereas the primary participants (the peer-educators) are referred to as “youth researchers”.

The Cornell facilitators introduced the concepts and techniques (described in detail later) of Participatory Action Research (PAR) in the context of the RTK project during the first RTK orientation workshop for youth researchers and project managers. Young people's roles as researchers, not educators, were emphasized during the workshop. With support from adults, youth researchers developed their research questions, as well as lists of priority groups that they had wanted to reach for youth-led PAR research. They recruited sharing team members through 
International Journal of Child, Youth and Family Studies (2012) 4: 396-408

churches, summer camps conducted by their youth organizations, programs for street/working children, and their own personal networks. The youth-led PAR research sessions took place one weekend at the residential setting. Youth researchers conducted research with sharing team members by using visual and youth-friendly PAR tools.

The purpose of this paper is to examine the effects on the "sharing team members" of their involvements with the "youth researchers" through analysis of data gathered during interviews conducted by university researchers with the youth. The effects of participation on the youth researchers themselves have been previously published (Goto, Pelto, Pelletier, \& Tiffany, 2010).

\section{Background}

In this section we briefly review the range of experiences that have been previously documented for participatory action research, particularly in projects that are directed toward youth. To date, these analyses have been undertaken from the perspective of scholars who have sought to understand the nature of participatory research projects. Such analyses provide important background for examining and interpreting the experiences of the youths we present in this paper.

Different degrees or modes of participation have been observed in participatory projects. A review of such projects reveals a wide diversity in the power relationships between external investigators and the community, including between adults and young people (Hart, 1997). Gibbon (2000) developed “modes of participation” in order to examine both the relationships between practitioners/researchers and local people/participants. Modes of participation, which vary from "manipulation" and "co-option" to "delegated power” and "collective action”, describe different types of relationships between local people and outsiders (Gibbon, 2000).

Power relationships between the community and outsiders can shape the mode of participation (Leurs, 1998). Outsiders may manipulate or impose their own agenda and hinder the quality, type, and sustainability of participation. In the case of young people's participation, Hart (1997) developed the concept of a "Ladder of Participation" to describe the relationships between children/youth and adults in projects. Degrees of participation vary from “manipulation”, “decoration”, and “tokenism” to “child-initiated, shared decisions with adults”. Hart revealed the different modes of participation in the case studies and argued that one cannot assume that young people are willing to participate until a commitment is made to value their contribution.

To date, the PAR literature describes a number of studies in which young people were involved essentially in the role of "informants", rather than being actively involved in generating and interpreting data as well as planning and implementing actions. A lack of clarity about roles and decision-making in PAR by different stakeholders in a project has been identified as an issue (Flicker, 2008). Another feature that affects our ability to interpret the literature on young people's experiences in PAR projects is that they are often treated as a homogeneous group, and the diversity within the group is not explored. Very few empirical studies have examined youths' experiences in relation to different modes of participation and 
International Journal of Child, Youth and Family Studies (2012) 4: 396-408

their distinct impacts.

\section{Methods}

\section{Study participants and data collection}

This qualitative study was conducted in a Caribbean country where the first author served as a technical advisor during the RTK project. Toward the end of the project we, university researchers, conducted in-depth, semi-structured individual interviews with 17 sharing team members, 21 youth researchers, and 12 adult project managers in order to understand their experiences in youth-led participatory action research.

The main objective of the interviews was to learn about sharing team members' experiences, including what went well, challenges encountered, experiences and perspectives they shared with the youth researchers, whether sharing team members gained something through their experience in PAR, and how sharing team members would like to use (or were already using) their experience, talents, and/or ideas with their peers to address the issues they identified in the context of RTK. Separate interview guides for sharing team members, youth researchers, and project managers were developed with support from UNICEF staff and the Cornell RTK consulting team.

All the participants gave oral consent and were guaranteed strict confidentiality. The consent procedures, as well as the interview protocols, were designed to enable them to feel comfortable, regardless of their personal life circumstances (e.g., living on the street, dealing with family conflicts, or being HIV-positive). All the youth researchers, 11 of the managers, and the majority of 10 of the sharing team members were interviewed twice to further probe their perspectives on sharing team members' participation in youth-led PAR. Interviews lasted between 30 and 90 minutes and they were tape recorded. The study protocol was reviewed and approved by Cornell University on Human Subjects.

The first author's role in providing ongoing technical assistance also enabled her to conduct in-depth participant observations. Numerous meetings and activities, such as youth-toyouth PAR training, were observed and documented, and field notes were developed based on her observations, reflections, and exploration of issues as they arose.

\section{Data Analysis}

The interviews were transcribed and coded for themes, based on the principles of grounded theory (Strauss \& Corbin, 1998). The transcripts and field notes developed by the first author were shared with and reviewed by the other authors. ATLAS/ti, a qualitative data analysis software program, was used for data analysis. Both pre-identified and newly identified themes were examined and compared across the interviews. In addition, preliminary findings based on the interviews were shared with UNICEF staff and some of the study participants in order to increase the rigor and trustworthiness of the results (Lennie, 2006). 
International Journal of Child, Youth and Family Studies (2012) 4: 396-408

\section{Results}

Four themes dominated the interviews and are particularly helpful for understanding factors associated with sharing team members' participation in the RTK project. We review them in the following sections, with an emphasis on the sharing team members' responses.

\section{Youth researchers' roles in fostering participation of "at-risk" youth}

During the interviews, young people repeatedly stated that everybody participated and elaborated on their perspectives about how the process encouraged participation. All sharing team members who were interviewed said that they felt "comfortable" because they were working with other youth. Sharing team members spontaneously identified various aspects of the youth researchers' actions that helped them to feel engaged and comfortable. These included efforts to ensure fair participation, informal settings with many icebreakers, and encouraging interactions with other sharing team members, all of which were viewed as factors that facilitated the creation of a comfortable environment. As the PAR research was conducted in a residential setting, the youth researchers and sharing team members spent time together after dinner talking or doing recreational activities, which also helped them to get to know one another better.

As previously reported (Goto et al., 2010) the youth researchers respected the local knowledge that emerged from sharing team members. Sharing team members discussed their thoughts, feelings, experiences, and knowledge about issues including drugs and violence in relation to their own lives. Project managers also recognized that young people understood so much about HIV/AIDS and knew how to use condoms.

Those young people know so much. They are street smart. They can educate others. (Project manager)

Thus, many sharing team members felt their opinions and experience were respected and appreciated by youth researchers and project managers during the PAR research, which made them feel "comfortable". Some sharing team members stated during their interviews that their experience of sharing their knowledge, ideas, and values made them feel good about themselves, and that learning built up their self-esteem. Furthermore, some of them mentioned that sharing their knowledge, ideas, and values with other youth helped them think about their own lives and the lives of others. One of the sharing team members said that the most rewarding experience for him in RTK was "to listen to different opinions, especially about people with HIV/AIDS".

\section{Youth researchers' roles as educators}

Youth researchers' roles as educators also appeared to support the active participation of the sharing team members. The sharing team members felt they benefited from the information they gained during the PAR research sessions. Although the primary purpose of the PAR sessions with the sharing team members was to identify issues for future actions and contextual factors that needed to be taken into consideration in the development of actions, the interviews with the sharing team members revealed that from their perspective the sessions also had a direct educational component. This was especially the case among youth whose motivation to 
International Journal of Child, Youth and Family Studies (2012) 4: 396-408

participate in the PAR research was to learn about HIV/AIDS issues. During the interviews, they stated that if they hadn't been part of the RTK, they wouldn't have had other means for gaining access to information about HIV/AIDS. All the sharing team members said that they had learned a great deal about HIV/AIDS during the PAR process. In addition, the word "knowledge" was used in a broad way across our interviewees.

It [knowledge] is not just information. It's about life skill sessions, self-esteem building, personal development. So, it's not information in a vacuum. It is information about everything, life skill, self-esteem, everything because all of them affect their behavior. (Project manager)

Related to the matter of education, the youth researchers noted the benefits of youth-to-youth communication. For example, one of them said:

Young people are more comfortable getting information from other young persons because when trained persons come, they can be judgmental. We have professionals that are very judgmental. "You should not be having sex, you should go to church." It is better to train young persons. (Youth researcher)

The knowledge about HIV/AIDS that the sharing team members gained through PAR seemed to provide them with confidence in becoming educators themselves. Most of them mentioned that they planned to share or were sharing what they learned in RTK with their peers and/or family members.

I can use my knowledge at school. Also I could use it to teach others of my friends. We have student counselors at my school and I am one of them. I could use it with the students I see or to teach the other counselors so they can help other students. (Sharing team member)

Some sharing team members focused on the link between knowledge, self-confidence, and the feeling of wanting to help others.

In RTK, we learned a lot, and it makes you feel like you can become a good person. I don't want my friends to get HIV/AIDS. (Sharing team member)

If you have knowledge, you have self-confidence. You feel better about yourself. You don't feel shy. So, you can go out and say boldly if you have factual information. (Sharing team member)

In sum, we learned from the interviews that many, of not most, of the sharing team members participated in PAR in order to learn more about HIV/AIDS. They perceived that gaining factual knowledge, especially biomedical knowledge about HIV/AIDS, helped them develop self-confidence and self-esteem. 
International Journal of Child, Youth and Family Studies (2012) 4: 396-408

\section{The uses of visual and youth-friendly research methods focusing on critical thinking}

Both youth researchers and sharing team members raised the idea that the visual and youth-friendly PAR tools that were employed by the project fostered dialogue among youth and enhanced participation levels. Some of the project managers stated that the PAR approach provided youth who were not inclined to reading and writing or those who were not outspoken with the opportunity to articulate their ideas through PAR tools, such as drawing and mapping. One sharing team member noted:

The most rewarding experience, for me, was drawing. It was fun. I loved the group work. Everybody was contributing to it. (Sharing team member)

Some youth researchers noted that tools such as "condom mapping” could stimulate a further discussion when the sharing team members presented their maps in front of the others.

At first, they [sharing team members] think mapping is about drawing roads. But, when we explain to them, like, "you know, mapping is to find out more than one thing," what they [sharing team members] are saying is, "OK, it is hard to get condoms at the convenience store, at the gas station.” But they [sharing team members] start thinking, "Why? Why is it?" And then, they feel like, if they [store employees] are older persons, they will want to question you at first, and younger persons, they are more open.

(Youth researcher)

Through active participation by the sharing team members, youth researchers learned that street condom vendors, instead of professional health service providers, actually could teach others how to use a condom. They also discovered that their organizations needed to sensitize the service providers for program changes.

Another discovery from the analysis was that the process of sharing values, ideas, and experiences led some of the sharing team members to think about others and to understand what others are going through. Some sharing team members explicitly connected the sharing experience, their acquired knowledge about HIV/AIDS and self-confidence with the feeling of wanting to help others. Although this cannot be generalized to all the sharing team members in RTK, individual actions such as talking to friends and family about HIV/AIDS, described by some of the sharing team members, were apparently sparked by newly gained knowledge, selfconfidence, awareness of others, and the desire to help others.

\section{Youth-led PAR training by an egalitarian organization}

In many cases the role of sharing team members remained that of "research participants". Their subsequent action after their PAR experience was limited to talking to friends and family about HIV/AIDS - communicating at the individual level. On the other hand, the youth researchers from Teen AIDS Network (pseudonym), one of the participating youth organizations, perceived PAR as "an empowering process" and believed that anybody could become a researcher. They asserted that the researchers' role should not be confined only to "selected youth” and they sought ways to entice sharing team members into greater involvement. 
International Journal of Child, Youth and Family Studies (2012) 4: 396-408

Specifically, they conducted youth-led PAR training, which provided the sharing team members, who were initially research participants or informants, with the opportunity to become "PAR researchers". Thus, the youth-led PAR training significantly contributed to redefining the lines between "the researchers" and "the researched" among youth, and facilitated individuals' awareness of their own capabilities as researchers and change agents.

Youth leaders target marginalized people. I don't think it should be like that. Get them to work as some of the youth leaders. I believe that they [marginalized people] enjoy facilitating. (Youth researcher)

The continued and enhanced participation of sharing team members as PAR change agents at Teen AIDS Network demonstrated the power of youth-led PAR training to contribute to sustainability and longer-term changes based on PAR activities. Sharing team members who joined Teen AIDS Network shared their excitement of becoming PAR researchers.

You can talk to people in the community, friends, family, to teach them about HIV/AIDS and safe sex. (Sharing team member)

The opportunity to engage in more PAR activities was not available to all sharing team members involved in the RTK project described in this report. Some organizational cultures were not open to the engagement of at-risk and marginalized youth as change agents. Many youth researchers, sharing team members, and project managers from youth organizations other than Teen AIDS Network believed that only "selected youth" should represent the other youths, and that this was sufficient to ensure that a "youth voice” would be heard. Several sharing team members expressed disappointment during their interviews that they hadn't been invited to take part in RTK activities, other than the residential sessions where they served primarily as informants. Some specifically stated their frustration at not becoming PAR researchers involved in ongoing RTK activities and in other community change efforts. The subtle power issue regarding who was expected to become a change agent and who was to remain a research "informant" was, to a large degree, obscured in RTK due to the general emphasis on "youth participation” and the assumption that youth researchers could represent "young people”.

\section{Discussion}

The PAR literature describes a number of studies in which young people participated in research only as "informants" not as "researchers". These are cases where their involvement was limited to brief episodes, rather than providing longer-term opportunities to develop their capabilities and sense of voice (Prilleltensky, Nelson, \& Peirson, 2001). To the best of our knowledge, RTK is one of the few projects that fostered youth-led PAR training and one of the few contexts in which the dynamics of youth-led PAR involving other youth have been examined (Maglajlic \& Tiffany, 2006).

The current study suggests that effectively introduced PAR tools may have not only enhanced the participation of sharing team members, but also may have facilitated individual actions by sharing team members. A primary finding from the interviews with the community youth who were reached by the youth leadership is that the experience of serving as sources of 
information in a participatory action research project was one in which they felt their opinions and life experiences were respected and appreciated. According to their own perceptions and reflections, this had a number of effects: It furthered self-confidence; it made them feel "comfortable" and it helped them to feel "good about themselves". This finding corresponds to Hart's (1997) arguments about the association of participation and self-confidence in PAR activities with children and youth.

The results also suggest that the participation of sharing team members was enhanced through youth-to-youth communication about HIV/AIDS information. Sharing team members enjoyed learning about information related to HIV/AIDS from other youth in a safe environment, instead of from parents or health authorities. In addition to gaining factual knowledge, especially biomedical knowledge about HIV/AIDS, this knowledge acquisition also contributed to the development of self-confidence and self-esteem. Thus, PAR was not only a vehicle for effective discussion and dissemination of information related to HIV/AIDS, but was also a source of personal development.

In addition to gaining factual information about HIV/AIDS, the results suggest that sharing team members were able to critically reflect on their lives in relation to HIV/AIDS using PAR as an opportunity for dialogue with their peers. Youth researchers created contexts where sharing team members could share their experiences, ideas, and values with other participants. In Freire's pedagogy, to know means to intervene in one's reality (Freire, 1973). It can be hypothesized that dialogue and critical reflection that contextualized the factual information about HIV/AIDS helped the young people to internalize it, to understand what it meant for them, and to act upon their newly-found knowledge.

As other studies suggest (Chambers, 1994; Hart, 1997), our study found that PAR can be accessible to young people with diverse backgrounds and characteristics. In RTK, PAR provided young people with the opportunity to articulate their ideas through drawing, mapping, and other tools that do not require strong literacy or verbal communication skills. Similar to "edutainment" approaches used in a study for HIV prevention in Thailand (W. Fongkaew, K. Fongkaew, \& Suchaxaya, 2007), our study shows that peer education using participatory tools holds potential for health education.

While the interviews with both youth researchers and sharing team members revealed many positive aspects of youth-led participatory action, the study also illuminates many of the challenges faced while implementing participatory projects. The PAR tools that were employed by the youth researchers did not foster participation by all sharing team members. This may be due to inexperience on the part of the youth researchers about how to foster and promote critical discussions that lead to new insights on the part of participants. Our observations of the sessions suggest that the youth researchers themselves were more comfortable when the discussion emphasized biomedical aspects of the issues rather than social aspects.

Our study also addressed power relations within the community of youth that may have influenced the modes of participation in the RTK project. Heterogeneity of the community has been identified as a factor affecting participation of certain groups within the community (Leurs, 
1998). There exist power imbalances of access, information, class, and expertise (e.g., literacy) among different groups, which may influence modes of participation.

The issue of differential participation in community health projects has been a subject of continuing concern. There is evidence that health interventions tend to reach those who are better off, better motivated, and better educated (Gillies, 1998). In our study, the youth peer educators who became the youth researchers probably fall into the category of better educated and certainly better motivated. It is difficult to determine, with the data we have available, whether the sharing team members fully represent the most marginalized youth, but there is reason to believe that many, if not most, were living in difficult circumstances. Nonetheless, it is essential to remain sensitive to the fact that intervention strategies based on practitioners' preconceived notions of "marginalized youth" may neglect heterogeneity in youth and reach only a small number of them. Thus, "youth participation" must be more clearly defined and monitored over the course of a program, especially as it is a central concept in youth development and in HIV/AIDS prevention among youth.

\section{Conclusion}

The importance of youth participation in interventions that address issues that affect them is increasingly recognized, as is the idea that youth peer educators are important change agents (Powers \& Tiffany, 2006; Maglajlic \& Tiffany, 2006). In this study we sought to examine the effects on youth who were at risk of exposure to HIV/AIDS of being involved in a youth-led participatory action research project. Based on the comments and points they made during the course of in-depth interviews, we conclude that the benefits of participation went beyond the acquisition of knowledge to include positive effects on self-confidence and, in some cases, an interest in reaching out to others.

Moving from a focus on the community participants to the youth leadership, we suggest that the PAR approach employed by one of the community groups, the Teen AIDS Network, can serve as a model for reaching youth who otherwise would not benefit from conventional health interventions. The RTK project provided youth with the opportunity to familiarize themselves with the concept of PAR and to modify modes of participation in their PAR approach. However, effective uses of PAR at the organizational level may not take place unless the project provides enough support for the possibly slow process of learning about and developing expertise in conducting PAR (Maglajlic \& Tiffany, 2006). In RTK, sharing team members from Teen AIDS Network had the opportunity to receive youth-led PAR training to become change agents and subsequently take action collectively. This relied on the emergence of a youth-adult partnership (Zeldin, Petrokubi, \& Camino, 2008) and demanded support from project managers, UNICEF officers, and Cornell researchers. Apart from the youth who were engaged with the Teen AIDS Network, the sharing team members who were involved in the other RTK teams did not have the same supports and opportunities for continued engagement in health programs. Paul (1998) has argued that one to two years are required for individuals to obtain sufficient understanding and self-confidence to become fluent in using participatory tools for effective collective action and community change. Nurturing young people to become "PAR change agents" requires a longer time frame, an understanding of the developmental needs of youth, and commitment at the organizational and program levels as well as support from the donor community. 
Figure 1 summarizes the process of sharing team members becoming active change agents as youth PAR researchers. We conclude that the use of youth-led PAR with other youth in specific organizational contexts can promote the sustainability of the PAR approach as well as young people's empowerment through health promotion projects. At the same time, we recognize significant challenges in the application of youth-led PAR, particularly with respect to follow-up interventions and sustainability within organizations that do not yet possess a strong foundation for promoting youth empowerment. Academic researchers and practitioners who are committed to PAR principles play important roles in defining and clarifying "youth" participation, as well as in facilitating linkages between young people’s needs and health interventions, and in developing organizational contexts that are hospitable to young people as they discover their capabilities as change agents.

Figure 1: From informants to change agents: Empowerment process of sharing team members

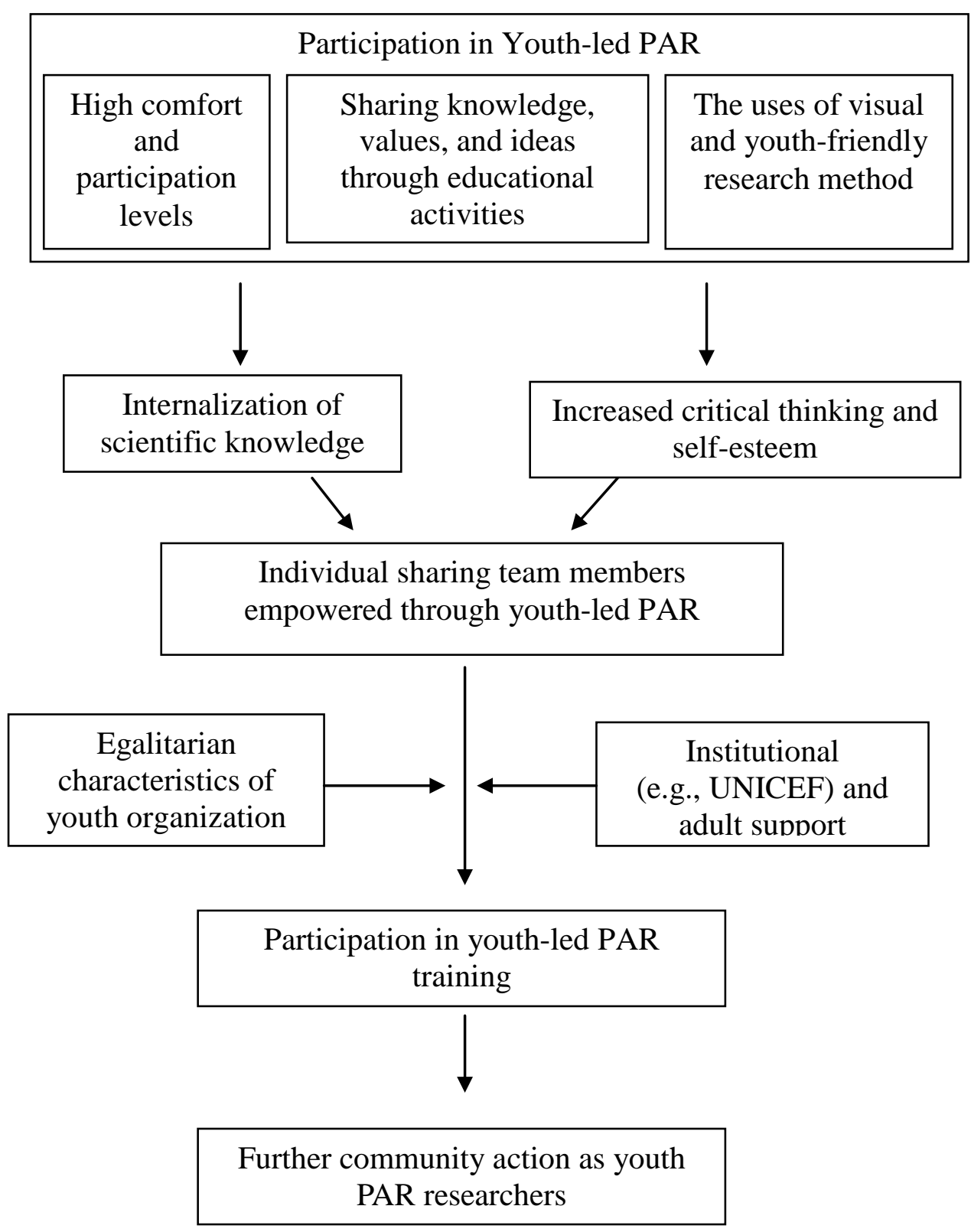




\section{References}

Blackburn, J., \& Holland, J. (1998). Who changes? Institutionalizing participation in development. London: Intermediate Technology Publications.

Chambers, R. (1994). The origins and practice of participatory rural appraisal. World Development, 22(7), 953-969.

Flicker, S. (2008). Who benefits from community-based participatory research? A case study of the positive youth project. Health Education \& Behavior, 35(1), 70-86.

Fongkaew, W., Fongkaew, K., \& Suchaxaya, P. (2007). Early adolescent peer leader development in HIV prevention using youth-adult partnership with schools approach. Journal of the Association of Nurses in AIDS Care, 18(2), 60-71.

Freire, P. (1973). Education for critical consciousness. New York: Seabury Press.

Gibbon, M. (2000). Partnerships for health: A way of working with women’s groups to improve community health in rural Nepal. In A. Cornwall, H. Lucas, \& K. Pasteur (Eds.), Accountability through participation: Developing workable partnership models in the health sector. IDS Bulletin, 31(1), 57-63.

Gillies, P. (1998). Effectiveness of alliances and partnerships for health promotion. Health Promotion International, 13(2), 99.

Goto, K., Pelto, G., Pelletier, D., \& Tiffany, J. (2010). “It Really Opened My Eyes”: The effects on youth peer educators of participating in an action research project. Human Organization, 69(2), 192-199.

Green, L. W., George, M. A., Daniel, M., Frankish, C. J., Herbert, C. P., Bowie, W.R., et al. (2003). Guidelines for participatory research in health promotion. In M. Minkler \& N. Wallerstein (Eds.), Community-based participatory research for health (pp. 419-428). San Francisco: Jossey-Bass.

Hart, R. (1997). Children's participation: The theory and practice of involving young citizens in community development and environmental care. New York: EarthScan.

Kemmis, S., \& McTaggart, R. (2000). Participatory action research. In N. K. Denzin \& Y. S. Lincoln (Eds.), Handbook of qualitative research (2nd ed., pp. 567-605). Thousand Oaks, CA: Sage Publications.

Lennie, J. (2006). Increasing the rigour and trustworthiness of participatory evaluations: Learning from the field. Evaluation Journal of Australasia, 6(1), 27-35. 
International Journal of Child, Youth and Family Studies (2012) 4: 396-408

Leurs, R. (1998). Current challenges facing participatory rural appraisal. In J. Blackburn \& J. Holland (Eds.), Who changes? Institutionalizing participation in development (pp. 124134). London: Intermediate Technology Publications.

Maglajlic, R., \& Tiffany, J. (2006). Participatory action research with youth in Bosnia and Herzegovina. Journal of Community Practice, 14(1/2), 163-181.

Minkler, M., \& Wallerstein, N. (2003). Introduction to community based participatory research. In M. Minkler \& N. Wallerstein (Eds.), Community based participatory research for health (pp. 3-26). San Francisco: Jossey-Bass.

Paul, B. (1998). Scaling-up PRA: Lessons from Vietnam. In J. Blackburn \& J. Holland (Eds.), Who changes? Institutionalizing participation in development (pp. 18-22). London: Intermediate Technology Publications.

Powers, J. L., \& Tiffany, J. S. (2006). Engaging youth in participatory research and evaluation. Journal of Public Health Management and Practice, 12(6) (Nov./Dec. Supplement), S79-S87.

Prilleltensky, I., Nelson, G., \& Peirson, L. (2001). The role of power and control in children's lives: An ecological analysis of pathways toward wellness, resilience and problems. Journal of Community \& Applied Social Psychology, 11(2), 143-158.

Strauss, A. L., \& Corbin, J. (1998). Basics of qualitative research: Techniques and procedures for developing grounded theory (2nd ed.). Thousand Oaks, CA: Sage Publications.

United Nations International Children's Emergency Fund (UNICEF). (2002). What every adolescent has a right to know. New York: Author.

Zeldin, S., Petrokubi, J., \& Camino, L. (2008). Youth-adult partnerships in public action: Principles, organizational culture and outcomes. Washington, D.C.: The Forum for Youth Investment. Retrieved June 11, 2011 from http://www.forumforyouthinvestment.org/files/YouthAdultPartnerships.pdf 